\title{
Awake nasotracheal fiber-optic intubation in a patient with severe gunshot injury to the face: Case Report
}

Kabugi J.G., MBChB,MMed,Fell (Cardiac Anesthesia), Okelo S. MBChB,MMed. Department of Anesthesiology and Critical Care Medicine, Moi Teaching \& Referral Hospital, Eldoret, Kenya. Request for reprints to Dr J.G. Kabugi .P.0. Box 5978 -30100, Eldoret, Kenya. E-mail: jkabugi@gmail.com

\section{Summary}

Facial trauma significantly increases the difficulty in airway management in the emergency department or during the provision of anesthesia for surgery. We present airway management in a patient with severe facial trauma that necessitated awake nasotracheal fiber-optic intubation for safe induction of anesthesia.

\section{Introduction}

Trauma to the face presents considerable challenges to the anesthesiologist especially when airway management is required as an emergency (1-5). Difficult airway management skills are necessary in the safe and timely control of ventilation in these desperate patients.

We describe the management of a patient with severe gunshot injury to the face requiring surgical debridement of burn injured tissue and reconstructive surgery in a staged fashion.

\section{Case report}

A 49year old gentleman presented to our hospital four days after being shot in the face at point blank range with an AK 47 assault rifle. During this period, he had not eaten anything due to his injuries. He was also not able to vocalize although he could phonate. His injuries included Le Forte III fracture with laceration of lower lip and loss of upper lip, fracture maxilla with loss of its anterior part together with part of the hard palate and columella, lacerated nostril floor with tissue loss, comminuted fracture mandible with loss of incisors and premolars, and degloving injury of the posterior part of the tongue (Fig. 1).He required extensive surgical debridement and later, reconstructive surgery.

A candid explanation of his airway management was given to him including the fact that this was to be done while he was awake. He was instructed to take deep breaths and to swallow any secretions he felt in his pharynx. Surgical support for possible emergency tracheotomy was on standby. Oxygen was administered by insufflation as it was not possible to place a facemask on him. After premedication with $0.4 \mathrm{mg}$ of atropine intravenously and $2 \mathrm{mg}$ of midazolam, $2 \%$ lidocaine was used to anesthetize his nasal and nasopharyngeal mucosae using a nozzled canister with lidocaine. Trans-tracheal injection of $3 \mathrm{~mL} 2 \%$ lidocaine was used to achieve tracheal anesthesia while a superior laryngeal nerve block was used to achieve glottic anesthesia.

A well lubricated $6.5 \mathrm{~mm}$ endotracheal tube was loaded onto a fiber-optic bronchoscope and the lubricated bronchoscope passed through the right nostril. The cords were visualized with some difficulty and the probe passed into the trachea. The tube was railroaded into the trachea and anesthesia induced with intravenous thiopental before cuffing the endotracheal tube. Visualization of the carina confirmed correct tracheal tube placement and surgery commenced. The patient required similar airway management twice for reconstructive surgery.

\section{Discussion}

The first fiber-optic nasotracheal intubation was reported by Peter Murphy,in 1967 on a patient with Still's disease using a flexible fiber-optic choledochoscope (1) The technique is indicated in patients with difficult airways in whom losing the airway following induction of anesthesia is deemed dangerous or fatal.

Successful awake fiber-optic intubation requires con- 
siderable experience in non-awake techniques. Skilled assistance, a well-informed patient and readiness for the creation of a surgical airway are necessary prerequisites. Appropriate medication should be readily available and drawn up for immediate use. Anesthetizing the airway well enough for the patient to tolerate awake intubation is usually a challenge. This, therefore, may necessitate the cautious use of conscious sedation, mild sedation or just anxiolysis (2).

The nasal and nasopharyngeal mucosae are anesthetized using topical lidocaine using a nozzled applicator. The patency of the nares may be improved using topical vasoconstrictors such as phenylephrine or oxymetazoline. The oropharynx can be anesthetized by asking the patient to gargle and swallow $4 \%$ viscous lidocaine. An end expiration trans-tracheal injection of $4 \%$ lidocaine results in a deep breath followed by a cough that evenly spreads out the anesthetic within the trachea (3).

It may not reliably anesthetize the glottis but this is easily achieved using a superior laryngeal nerve block at the greater cornu of the hyoid bone.

Once the airway is well anesthetized, there is no protection against aspiration of oropharyngeal or gastric secretions making this stage dangerous in patients who may be having a full stomach. Premedication with a gastrokinetic agent such as metoclopramide is helpful in reducing the risk of aspiration of gastric contents. A proton pump inhibitor may also be used to increase the $\mathrm{pH}$ of gastric contents. An antisialogogue like glycopyrrolate will reduce secretions and facilitate glottic visualization (4).

The flexible bronchoscope is passed into the nostril, nasopharynx and hypopharynx to visualize the vocal cords. The bronchoscope is then passed into the trachea and confirmed to be correctly placed by the visualization of tracheal rings. Further advancement of the scope will reveal the carina. The Endotracheal tube is then railroaded into the trachea and anesthesia immediately induced even before inflating the endotracheal tube cuff. Further confirmation of correct endotracheal tube placement is required using capnography and a second flexible bron- choscopic visualization of the carina.

Proficiency and experience in awake fiber-optic airway management is a necessary addition to the anesthesiologist's armamentarium of difficult airway management options (5).

\section{References}

1. Murphy P. A fibre-optic endoscope used for nasal intubation. Anaesthesia 1967; 22:489-491.

2. Wheeler M, Ovassapian. Fiberoptic endoscopy-aided techniques. In Hagberged: Benumof's Airway Management, 2nd ed. Mosby Elsevier; 2007.

3. Webb AR, Fernando SS, Dalton HR, et al. Local anaesthesia for fibreoptic bronchoscopy: Transcricoid injection or the "spray as you go" technique?. Thorax 1990; 45:474-477

4. Benumof JL: Management of the difficult adult airway with special emphasis on awake tracheal intubation. Anesthesiology 1991; 75:1087-1110.

5. Caron G, Paquin R, Lessard MR, et al. Submental endotracheal intubation: an alternative to tracheotomy in patients with midfacial and panfacial fractures. J Trauma. 2000;48(2):235-40. 